Rev. Elev. Méd. vét. Pays trop., 1979, 32 (1) : 25-32

\title{
Brucella abortus d'origine bovine au Sénégal : identification et typage
}

\author{
par J. M. VERGER (*), M. GRAYON (*), M. P. DOUTRE $\left({ }^{* *}\right)$ et F. SAGNA (**)
}

\begin{abstract}
RÉSUMÉ
Cent quatre-vingt une souches de Brucella d'origine bovine, isolées au Séné gal, de 1976 à 1978, ont été identifiées par l'ensemble des épreuves recommandées par le sous-Comité de la Taxonomie de ce genre bactérien. Leurs caractères sont, pour l'essentiel, conformes à la définition de l'espèce Brucella abortus au sein de laquelle 180 souches appartiennent au biotype 3 et une au biotype 1 .

Deux caractères inhabituels pour cette espèce distinguent toutefois les souches sénégalaises: leur réponse négative - à une exception près - à l'épreuve de l'oxydase et leur profil moyen d'oxydation métabolique modifié au niveau de 4 des substrats conventionnels ( $L$-asparagine, L-arabinose, $D$ galactose et D-xylose). Ces deux caractères originaux sont discutés d'un double point de vue, épidémılogique et taxonomique.
\end{abstract}

Le genre Brucella est subdivisé en 6 espèces (10). Cinq sont présentes en Afrique : $B$. abortus, B. melitensis, B. suis, B. ovis (18) et B. canis (21). La rareté des articles consacrés au typage limite malheureusement la connaissance de la nature et de la fréquence des biotypes correspondants.

En ce qui concerne le Sénégal, les données publiées par CHAMBRON, en 1965 (3), portent sur un nombre trop restreint de souches et n'intègrent pas les propositions du SousComité de la Taxonomie des Brucella, alors récemment créé, sur la différenciation au sein des espèces de ce genre bactérien (15).

L'application de ces propositions à l'étude de 181 souches de $B$. abortus d'origine bovine isolées dans ce pays, de 1976 à 1978, fait l'objet du présent travail.

\section{MATÉRIEL ET MÉTHODES}

\section{Souches bactériennes}

Les 181 souches isolées à la suite de différentes tournées accomplies dans le sud du Séné-

(*) Station de Pathologie de la Reproduction, Centre de Recherches de Tours, I. N. R. A., Nouzilly, 37380 Monnaie, France.

(**) Laboratoire national de l'Elevage et de Recherches vétérinaires, I. S. R. A., B. P. 2057, Dakar-Hann, République du Sénégal. gal (Sine-Saloum, Sénégal-Oriental, Haute, Moyenne et Basse-Casamance), de 1976 à 1978 , sont toutes d'origine bovine et proviennent chacune d'un animal différent (carte). Tous les troupeaux visités appartiennent à la race Ndama. Les isolements ont été obtenus à partir de liquides de ponction d'hygromas mis en culture sur milieu «Brucella agar modifié », additionné de polymyxine, bacitracine et cycloheximide (mélange P. B. C. lyophilisé) (*).

Après 4 jours d'incubation à $37^{\circ} \mathrm{C}$, sous atmosphère convenable de $\mathrm{CO}_{2}$ (GASPAC Anaerobic System $\left({ }^{*}\right)$ ), l'examen morphologique et microscopique permet de reconnaître, sur les boîtes de milieu sélectif, les colonies de Brucella. Celles-ci sont repiquées sur des pentes de "gélose trypticase soja » (*) supplémentée à 1 p. 1000 d'extrait de levure et, après incubation, les tubes de culture sont envoyés à Nouzilly pour identification et typage.

\section{Méthodes d'identification}

- La détermination du genre Brucella repose sur les méthodes bactériologiques simples (tabl. l) recommandées pour le diagnostic différentiel avec les groupes de bactéries à Gram négatif pouvant prêter à confusion $(1,10)$.

(*) BIOMERIEUX : Marcy l'Etoile, 69260 Charbonnières-les-Bains, France. 


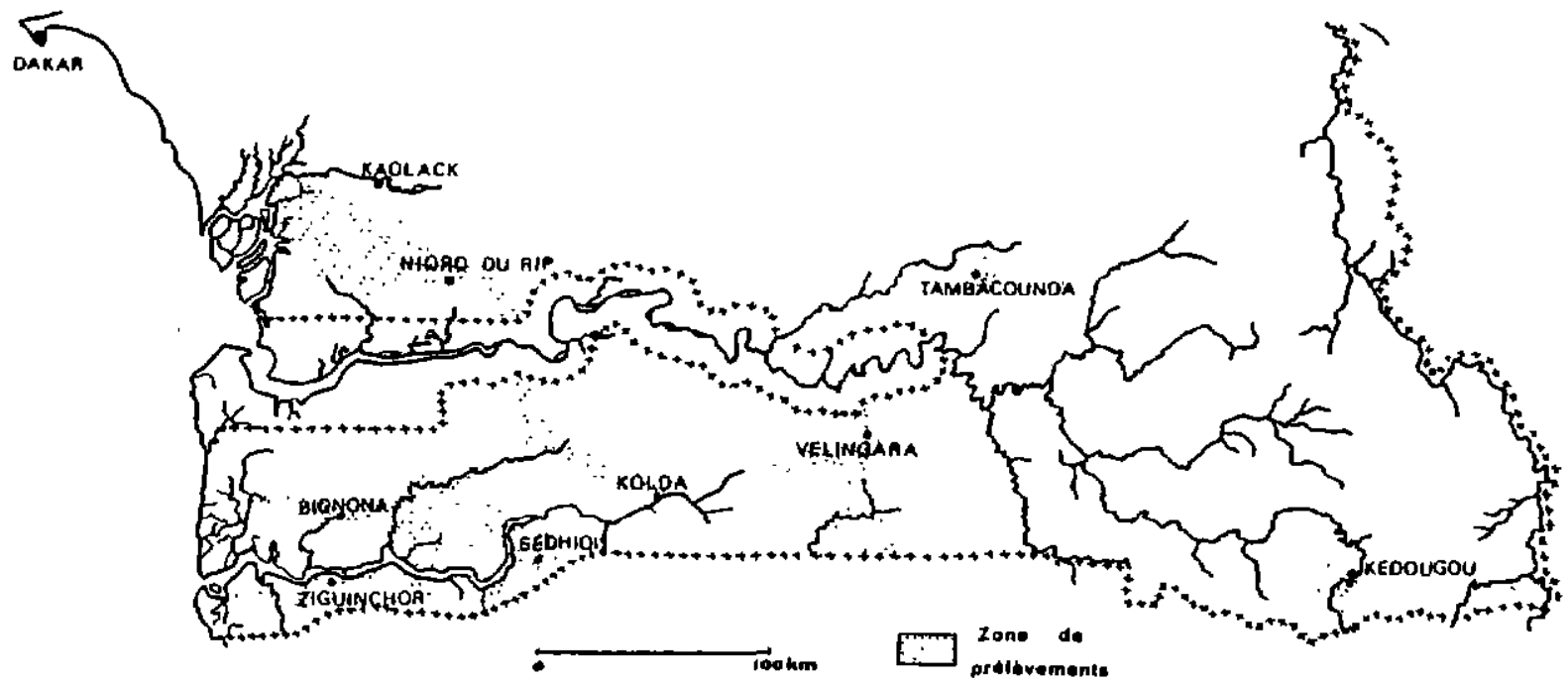

Fig. 1. - Origine géographique des 181 souches de Brucella abortus d'origine bovine isolées au Sénégal.

Tableau I. - Caractérisation de 181 souches de Brucella d'origine bovine isolées au Sénégal, par les épreuves courantes d'identification des bactéries à Gram négatif.

\begin{tabular}{|c|c|}
\hline Caractères & Résultats \\
\hline $\begin{array}{l}\text { Morphologie cellulaire } \\
\text { Coloration de Gram } \\
\text { Mobilité } \\
\text { Fermentation du lactose sur Mac Conkey agar } \\
\text { Hémolyse sur gélose sang } \\
\text { Acidification du glucose en eau peptonée, rouge de phénol } \\
\text { Catalase } \\
\text { Oxydase } \\
\text { Uréase } \\
\text { Indole } \\
\text { Réduction des nitrates } \\
\text { Citrate (Simmons) } \\
\text { Rouge de méthyle (R.M.) } \\
\text { Voges-Proskauer (V.P.) }\end{array}$ & $\begin{array}{c}\text { Coccobaciles non sporulés, non encapsulés } \\
- \\
- \\
- \\
- \\
180-, 1+ \\
+ \\
+ \\
- \\
-\end{array}$ \\
\hline
\end{tabular}

L'épreuve de l'oxydase est pratiquée, pour chaque souche, suivant deux techniques: la première est celle classique de KOVACS ; la seconde utilisant, comme réactif, l'hydrochlorure de $\mathrm{N}$-diméthyl-paraphénylène-diamine, permet, en versant celui-ci à la surface du milieu solide de culture, de tester rapidement l'homogénéité de la réaction de l'oxydase au sein d'une population de colonies bactériennes. Ces deux techniques sont décrites par LENNETTE, SPAULDING et TRUANT (6).

- Les épreuves courantes d'identification des espèces de Brucella et de leurs biotypes sont présentées dans le tableau II. Ce sont celles recommandées par le Sous-Comité de la Taxo- nomie des Brucella $(5,15)$ et décrites par ALTON, JONES et PIETZ (1).

- L'activité respiratoire vis-à-vis des 11 substrats indiqués sur le tableau III (métabolisme oxydatif) est déterminée pour 65 souches de l'échantillon. La méthode employée est décrite antérieurement $(11,19,20)$ et l'expression graphique des résultats (fig. 2) est conforme à celle proposée dans un précédent travail (22).

\section{RÉSULTATS}

Les souches sénégalaises sont caractérisées par de nombreuses réponses négatives aux épreuves bactériologiques courantes d'identification 
des bactéries à Gram négatif (tabl. I). Ceci est conforme au comportement habituel des Brucella. Le test de l'oxydase, négatif pour toutes les souches sauf une, mérite attention. Il sera discuté plus loin.

Les résultats des épreuves courantes de différenciation au sein du genre Brucella sont présentés dans le tableau II. Toutes les souches, de type morphologique smooth, sont lysées, aux

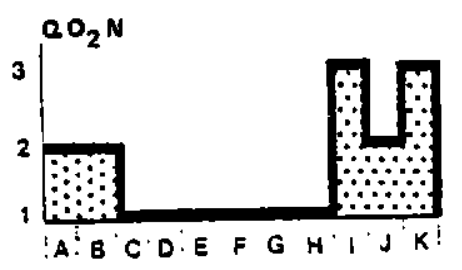

B. abortusisonigal)

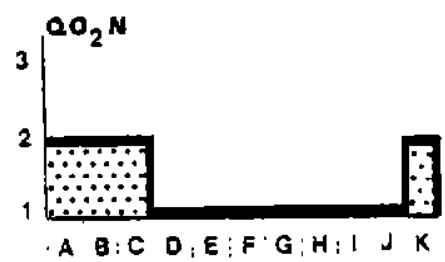

B. melitensis deux dilutions d'épreuve (DCE et $10^{4} \times$ DCE), par le bactériophage $\mathrm{Tb}$ spécifique de Brucella abortus. Les autres caractères différencient les biotypes :

- Cent quatre-vingt souches exigent l'addition de $\mathrm{CO}_{2}$ pour leur croissance, produisent de l' $\mathrm{H}_{2} \mathrm{~S}$, sont uniquement agglutinées par le sérum monospécifique ariti-abortus-suis (A) et poussent en présence de thionine aux 3 concen-

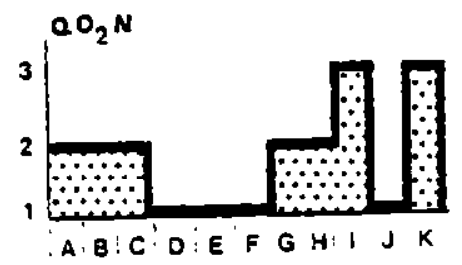

\section{B. abortus}

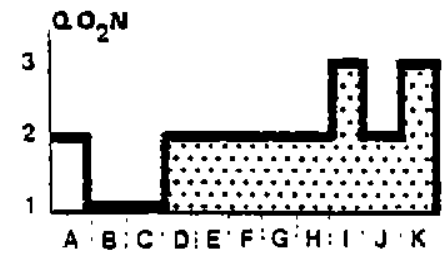

B. suis 1

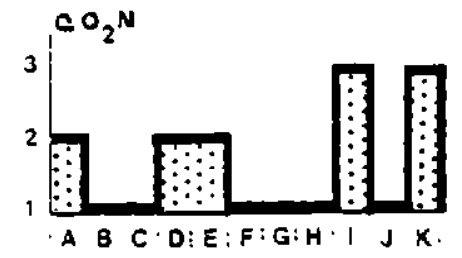

B. suis 3

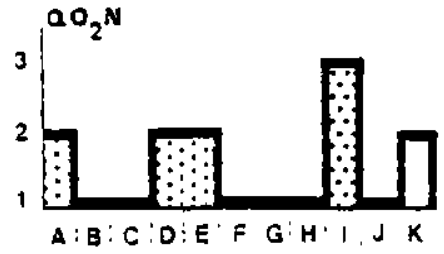

B. canis

Fig. 2. - Représentation graphique des résultats du métabolisme oxydatif : Profil métabolique moyen des souches de Brucella abortus d'origine bovine isolées au Sénégal.

Comparaison avec les profils métaboliques les plus probables pour les espèces et types de Brucella dont la présence a été signalée en Afrique.

Niveau $\mathrm{QO}_{2} \mathrm{~N}$ :

1. $\mathrm{QO}_{2} \mathrm{~N}$ moyen inférieur à 100

2. $\mathrm{QO}_{2} \mathrm{~N}$ moyen compris entre 100 et 300

3. $\mathrm{QO}_{2} \mathrm{~N}$ moyen supérieur à 300 .

Substrats :

$$
\begin{aligned}
& \mathbf{A}=\text { Acide L-glutamique } \\
& \mathbf{B}=\text { L-alanine } \\
& \mathbf{C}=\text { L-asparagine } \\
& \mathbf{D}=\text { L-arginine }
\end{aligned}
$$

$\mathrm{E}=\mathrm{DL}$-ornithine

$\mathbf{F}=$ L-lysine

$\mathrm{G}=\mathrm{L}$-arabinose

$\mathbf{H}=\mathbf{D}$-galactose

8. ovis

Surface pointillée : profil le plus probable quelle que soit la souche.

Surface claire : profil moyen de l'échantillon mais, en raison du grand intervalle entre les valeurs extrêmes observées, le substrat peut être oxydé à l'un ou l'autre des 3 niveaux. 


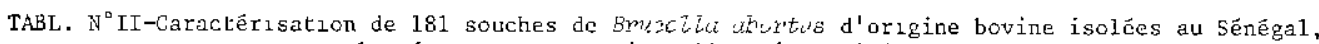
par les épreuves conventionnelles d'zdentification.

\begin{tabular}{|c|c|c|c|c|c|c|c|c|c|c|c|}
\hline \multirow{3}{*}{$\begin{array}{c}\text { Nombre } \\
\text { de } \\
\text { souches }\end{array}$} & \multirow{3}{*}{$\begin{array}{l}\text { oxyda- } \\
\text { se }\end{array}$} & \multirow{2}{*}{$\begin{array}{l}\text { Lyse jar le } \\
\text { plunge Tb }\end{array}$} & \multirow{3}{*}{$\begin{array}{l}\text { Exigence } \\
\text { en } \mathrm{CO}_{2}\end{array}$} & \multirow{3}{*}{$\begin{array}{l}\text { Production } \\
\text { de } \mathrm{H}_{2} \mathrm{~S}\end{array}$} & \multirow{2}{*}{\multicolumn{2}{|c|}{$\begin{array}{l}\text { digglitivalioil } \\
\text { par les sêrums } \\
\text { monospécıflques }\end{array}$}} & \multicolumn{3}{|c|}{$\begin{array}{c}\text { Croissance en prësence } \\
\text { de colorants }\end{array}$} & \multirow{3}{*}{ Brotype } & \multirow{3}{*}{ Espèce } \\
\hline & & & & & & & Thyonine & $\begin{array}{l}\text { Fuschine } \\
\text { basigue }\end{array}$ & 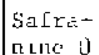 & & \\
\hline & & $\mid \begin{array}{c}\operatorname{DCS} . \\
\text { 111 }\end{array}$ & & & & $M$ & $\begin{array}{lll}10 & 20 & 40 \\
3 & \end{array}$ & $10 \quad 20$ & 100 & & \\
\hline 1 & - & + & + & + & + & - & $\begin{array}{lll}- & - & -\end{array}$ & $+\quad+$ & + & 1 & \\
\hline 1 & + & + & + & + & + & - & +++ & $+\quad+$ & + & & \\
\hline 179 & - & + & + & $T$ & + & - & +++ & + & + & 3 & 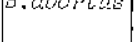 \\
\hline
\end{tabular}

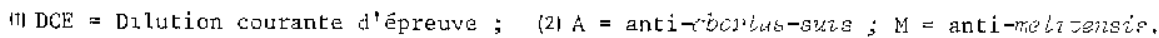

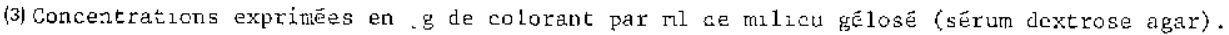

trations indiquées. Ces caractères définissent B. abortus biotype 3 .

- Une souche identique par ailleurs aux précédentes s'en distingue par sa sensibilité aux mêmes concentrations de thionine. Elle est, de ce fait, classée dans le biotype 1 de l'espèce.

Les résultats de l'épreuve de l'oxydase figurent aussi dans le tableau II. Une seule souche, appar- tenant au biotype 3, est positive. Toutes les autres, y compris la seule classée dans le biotype 1 , sont oxydase négatives: ce comportement est inhabituel pour l'espèce $B$. abortus.

Les résultats de l'étude du métabolisme oxydatif de l'unique souche du biotype 1 et de 64 parmi celles du biotype 3 -dont la seule oxydase positive - sont présentés dans le tableau III. Il apparaît que, pour les 65 souches, TABL. N ${ }^{\circ} \mathrm{III}$-Consommations d'oxygène $\left(\mathrm{QO}_{2} \mathrm{~N}\right)$, après 24 heures de culture sur m11eu "Trypticase Soy agar", de 65 souches de Bruceța abontwo d'origine bovine isolëes au Sénégal, en présence de diffẻrents substrats

\begin{tabular}{|c|c|c|c|c|c|c|}
\hline \multicolumn{2}{|r|}{ Biotype } & 1 & \multicolumn{4}{|c|}{3} \\
\hline \multicolumn{2}{|c|}{ Oxydase } & - & + & \multicolumn{3}{|c|}{-} \\
\hline \multicolumn{2}{|c|}{ Drmension de l'échantilion } & 1 & I & \multicolumn{3}{|c|}{63} \\
\hline \multicolumn{2}{|c|}{$\begin{array}{l}\text { Paramètres statistiques ou } \\
\text { valeurs Individuelles }\end{array}$} & $\mathrm{x} 1$ & $\mathrm{X} 1$ & W & $\overline{\mathrm{x}}$ & $\mathrm{S}$ \\
\hline \multirow{12}{*}{ 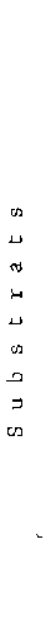 } & $0^{: 2}$ & 97 & 87 & $65-147$ & 97 & 18 \\
\hline & Acıde L-glutamique & 266 & 222 & $208-409$ & 279 & 39 \\
\hline & L-alaninc & 135 & 171 & $148-298$ & 225 & 31 \\
\hline & L-asparagine & 0 & 0 & $0-18$ & 2 & 4 \\
\hline & L-arginine & 37 & 53 & $39-132$ & 80 & 18 \\
\hline & DL-ornithine & 0 & 28 & $12-116$ & 47 & 23 \\
\hline & L-1ysine & 0 & 14 & $0-35$ & 15 & 9 \\
\hline & L-arabinose & 14 & 33 & $5-87$ & 45 & 17 \\
\hline & D-galactose & 13 & 15 & $2-102$ & 39 & 18 \\
\hline & D-ribase & 382 & 397 & $257-506$ & 391 & 54 \\
\hline & D-xylose & 165 & 173 & $102-275$ & 180 & 38 \\
\hline & Másoerythrıtol & 430 & 370 & 182-504 & 370 & 60 \\
\hline
\end{tabular}

$\because$ La respiration endogène (colonne 0) est soustraite et les valeurs sont exprimécs cr microlitres d'axygène consommé par milligrame d'azote bactérien et par heure $\left(10_{2} / \mathrm{mg} \mathrm{N} / \mathrm{h}\right)$.

Symboles utilisês : $W$, amplıtude ou intervalle entre les valeurs extrêmes observẻes ;

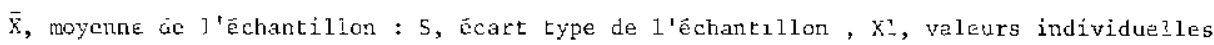


les valeurs d'oxygène consommé vis-à-vis des substrats indiqués sont homogènes et indépendantes $\mathrm{du}$ biotype et $\mathrm{du}$ caractère oxydase. L'expression graphique de ces résultats (fig. 2) montre que le profil métabolique moyen des souches sénégalaises ressemble au "profil le plus probable " défini pour l'espèce $B$. abortus. Ce profil spécifique est cependant altéré, chez les souches sénégalaises, au niveau de 4 substrats :

- la L-asparagine, le L-arabinose et le Dgalactose qui sont métabolisés au $1^{\text {er }}$ niveau $\left(\mathrm{QO}_{2} \mathrm{~N}\right.$ moyen inférieur à 100$)$ au lieu du deuxième ;

- le D-xylose qui est oxydé au niveau 2 $\left(\mathrm{QO}_{2} \mathrm{~N}\right.$ moyen entre 100 et 300$)$ au lieu du premier.

En dépit de ces variations, le profil d'oxydation métabolique des souches sénégalaises ne peut être confondu avec celui des espèces de Brucella autres qu'abortus.

\section{DISCUSSION}

L'identification et le typage des 181 souches sénégalaises n'ont pas soulevé de difficulté. Les caractères morphologiques, culturaux et biochimiques, présentés dans les résultats, sont conformes à ceux décrits pour le genre Brucella $(1,10)$ et le classement dans les biotypes 1 et 3 de $B$. abortus (tabl. II) ne souffre aucune ambiguité. Deux caractères inhabituels pour cette espèce méritent cependant discussion : la réponse négative à l'épreuve de l'oxydase et l'aspect original du profil d'oxydation métabolique (*).

\section{Caractère oxydase}

Le genre Brucella comprend 6 espèces. Quatre sont réputées oxydase positives: $B$. abortus, $B$. melitensis, $B$. suis et $B$. canis; $B$. ovis et $B$. neotomae sont caractérisées, au contraire, par des réactions négatives $(1,10)$. Toutes les souches de $B$. abortus, d'origines géographiques très diverses, que nous avons typées jusqu'à présent ont effectivement toutes répondu positivement à l'épreuve de l'oxydase. Le comportement négatif des souches sénégalaises -

(*) Une inhabituelle lenteur de croissance est également manifestée par toutes les souches sur milieu Trypticase Soy Agar. Cette autre particularité, plus subtile mais régulièrement observée, vaut d'être notée. sauf une $\left({ }^{*}\right)$ - est donc très inhabituel. STEEL, cependant, a observé que des souches de $B$. abortus (1 sur 12), B. melitensis (1 sur 6) et $B$. suis ( 4 sur 7 ) étaient oxydase négatives (17). Il n'est donc pas impossible que, pour ces trois espèces, la réponse à l'épreuve de l'oxydase soit négative plus souvent que ne le laissent supposer les articles d'identification et de typage de ces bactéries, où souvent d'ailleurs les résultats de ce test ne sont pas mentionnés.

Ces faits soulignent donc l'intérêt taxonomique et épidémiologique de la mise en œuvre systématique de l'épreuve de l'oxydase pour l'étude bactériologique des Brucella.

Le support biochimique de la différence de réponse des espèces ou souches de Brucella à l'épreuve de l'oxydase n'est pas connu. Cette méthode, basée sur l'aptitude de la chaîne respiratoire à oxyder un certain nombre de colorants, ou systèmes redox, n'est pas spécifique et ne permet pas, à elle seule, de définir les bases biochimiques de la réaction de l'oxydase (14). Une corrélation entre la présence d'un cytochrome c dans la partie terminale de la chaîne respiratoire et une réponse positive à l'épreuve de l'oxydase a pu être avancée pour certains groupes bactériens (Pseudomonas, Moraxella) (16). Ce n'est pas le cas pour le genre Brucella dont toutes les espèces, y compris $B$. ovis et $B$. neotomae oxydase négatives, contiennent du cytochrome c $(12,13,23)$.

\section{Profil d'oxydation métabolique}

MEYER et CAMERON, sur une gamme définie de substrats (acides aminés et glucides), ont établi que chaque espèce de Brucella est caractérisée par un schéma d'oxydation métabolique particulier $(8,9)$. Nous avons montré (22) que les consommations d'oxygène $\left(\mathrm{QO}_{2} \mathrm{~N}\right)$, vis-à-vis des substrats choisis, peuvent être schématiquement ramenées à 3 niveaux :

- Le niveau 1 réunit les substrats vis-à-vis desquels la consommation d'oxygène est régulièrement faible : $\mathrm{QO}_{2} \mathrm{~N}$ moyen inférieur à 100 ;

- Le niveau 2 correspond à une consommation modérée d'oxygène : $\mathrm{QO}_{2} \mathrm{~N}$ moyen compris entre 100 et 300 ;

- Le niveau 3, enfin, groupe les substrats pour lesquels la consommation d'oxygène est

(**) Isolée au village de Ndoumbouthie, près de Sokone (Sine-Saloum). Onze autres souches oxydase négatives ont été isolées dans le même village. 
constamment élevée : $\mathrm{QO}_{2} \mathrm{~N}$ moyen supérieur à 300.

La représentation graphique de cette distribution des substrats matérialise un profil métabolique caractéristique de chaque espèce de Brucella (fig. 2). Le choix des valeurs limites (100 et 300) tient compte à la fois des consommations moyennes $(\bar{X})$ et de la dispersion des valeurs individuelles (W), de telle sorte que le profil métabolique moyen, ainsi défini, est le plus probable pour n'importe quelle souche de l'espèce considérée.

L'espèce $B$. abortus est donc caractérisée (fig. 2) par l'oxydation probable des substrats :

- L-arginine, DL-ornithine, L-lysine et Dxylose au niveau 1 ;

- acide L-glutamique, L-alanine, L-asparagine, $L$-arabinose et $D$-galactose au niveau 2 ;

- D-ribose et méso-érythritol au niveau 3.

Les souches sénégalaises diffèrent donc de ce profil type au niveau de 4 substrats : la L-asparagine, le L-arabinose, le D-galactose et le Dxylose qui sont oxydés à un niveau inhabituel. Leur profil métabolique ressemble cependant à celui de $B$. abortus plus qu'à aucun des autres espèces du genre (fig. 2).

Les souches sénégalaises ne sont pas les seules, au sein de l'espèce $B$. abortus, à présenter une fluctuation de $\mathrm{QO}_{2} \mathrm{~N}$ habituel, au niveau d'un ou même de plusieurs substrats.

- La souche vaccinale B 19 oxyde de façon caractéristique l'aciđe L-glutamique et le mésoérythritol, respectivement aux niveaux 3 et 1 $(2,22)$.

- Nous avons également rapporté l'isolement de 4 souches de $B$. abortus biotype $1-$ isolées chez une vache et 3 mulots (Apodemus sylvaticus) originaires d'une exploitation agricole massivement infectée de brucellose bovine - qui, comme les souches du Sénégal, manifestaient une consommation, d'oxygène inhabituellement faible (niveau 1) vis-à-vis de la Lasparagine $(20)$. Ce caractère original avait permis en l'occurrence de mettre en évidence un lien épidémiologique certain entre les souches isolées des mulots et celle d'origine bovine.

L'intérêt du marquage épidémiologique de souches de $B$. abortus biotype 1 par un caractère métabolique inhabituel a été également rapporté, dans l'Etat du Minnesota (U.S. A.), par LUCHSINGER et collab. (7).

Des variations du profil métabolique type ont également été observées chez les autres espèces de Brucella. C'est le cas, en particulier, de sou- ches de $B$. suis biotype 3 isolées par COOK et collab. chez des rongeurs dans le North Queensland (Australie) (4).

Ces exemples, parmi d'autres, ont le mérite de souligner le double intérêt du métabolisme oxydatif chez les Brucella.

1) Les variations rapportées jusqu'à présent, au niveau d'un ou plusieurs substrats, n'altèrent pas fondamentalement l'aspect général du profil métabolique spécifique. Celui-ci est donc un bon critère de détermination de l'espèce au sein du genre Brucella.

2) Ces variations représentent des marqueurs épidémiologiques d'autant plus précieux que l'absence d'outils de marquage suffisamment fins (lysotypes, facteurs de résistance...) caractérise les Brucella.

\section{CONCLUSION}

L'analyse épidémiologique repose essentiellement sur des observations liées à des méthodes d'investigation propres au Laboratoire. Pour être efficace, elle doit faire appel à toutes les méthodes disponibles, y compris celles qui relèvent de l'identification et du typage bactériologiques. Vaine, en effet, serait la prétention de maîtriser une maladie infectieuse en ignorant l'agent responsable, en tant que tel, sous ses différents aspects que sont les espèces et leurs divers types.

C'est dans cet esprit que nous avons entrepris l'étude bactériologique des souches de Brucella isolées au Sénégal. La mise en évidence, sur celles-ci, de deux caractères inhabituels ayant valeur de marqueurs, illustre bien l'intérêt épidémiologique et taxonomique de l'identification et du typage des Brucella pour lesquelles, tant en Afrique que dans le reste du monde, la connaissance de la nature et la fréquence des différents types responsables de l'infection est encore trop fragmentaire.

Pour se limiter au continent africain, il serait souhaitable que l'ensemble des souches isolées par les chercheurs des différents laboratoires soient centralisées afin de parvenir à une connaissance taxonomique plus complète.

\section{REMERCIEMENTS}

Qu'il nous soit permis ici de remercier MM. Mamadou DIALLO (Directeur du Service de la Santé et des Productions Animales), 
Moustapha DIA, Serigne Mbaye DIALLO et Sidy NIANG, respectivement Inspecteurs régionaux à Kaolack, Tambacounda et Ziguinchor, les Chefs de secteurs départementaux : Alioune TOURE (Nioro du Rip), Boubacar NGOM (Foundiougne), Mamadou NDAO (Kédougou), Gnele KANOUTE (Vélingara), Lassana NDIAYE (Kolda), Amadou GUINDO (Sédhiou),
Mbacké FAYE (Ziguinchor), Bécaye DIALLO (Bignona), Moussa SANGARE (Oussouye) et les agents techniques Issakha DIOP (Vélingara) et Mamadou DIATTA (Kolda) dont l'action conjointe a permis de recueillir sur le terrain, dans d'excellentes conditions, le matériel biologique nécessaire à l'exécution du présent travail.

\section{SUMMARY}

\section{Brucella abortus isolated from cattle in Senegal : identification and typing.}

One hundred and eighty one strains of Brucella isolated from cattle in various areas of Senegal, during a two-year period from 1976 to 1978 , were examined by all methods recommended by the subcommittee on Taxonomy of the genus Brucella. All were found to have the same main characteristics that identify and define the species Brucella abortus. Of the 181 strains, all but one were biotype 3 ; the single different strain was biotype 1 .

However two unusual characters distinguish the senegalese strains from the main group of Brucella abortus : the oxidase test negative for all but one strains and the specific oxidative profile altered on four of the conventional substrates (L-asparagine, L-arabinose, D-galactose and D-xylose). These two original characters are discussed from an epidemiological and taxonomical point of view.

\section{RESUMEN}

\section{Brucella abortus de origen bovina en Senegal : identificación}

Se identificaron 181 cepas de Brucella de origen bovino, aisladas en Senegal de 1976 a 1978, mediante el conjunto de las pruebas recomendadas por la subcomisión de la taxonomía de dicho género bacteriano. Sus características son, en lo esencial, formadas con la definición de la especie Brucella abortus en el seno de las cuales 180 cepas pertenecen al biotipo 3 y 1 al biotipo 1 .

Sin embargo, las cepas senegalesas tienen dos características inhabituales : su reacción negativa, con excepción de una, a la prueba de la oxidasa y su perfil medio de oxidación metabolica modificado al nivel de 4 de los substratos convencionales ( $\mathrm{L}$-asparagina, L-arabinosa, D-galactosa y D-xylosa). Se discuten estas dos características desde los dos puntos de vista : epidemiologico y taxonómico.

\section{BIBLIOGRAPHIE}

1. ALTON (G. G.), JONES (L. M.), PIETZ (D. E.). $\mathrm{La}$ brucellose. Techniques de laboratoire, $2^{\mathrm{c}}$ éd. Genève, O. M. S., 1977.

2. BROWN (G. M.), LOVE (E. L.), PIETZ (D. E.), RANGER (C. R.). Characterization of Brucella abortus strain 19. Am. J. vet. Res., 1972, $33: 759-764$.

3. CHAMBRON (J.). La brucellose bovine au Sénégal. Rev. Elev. Méd. vét. Pays trop., 1965, 18 (I) : 19-38.

4. COOK (I.), CAMPBELL (R. W.), BARROW (G.). Brucellosis in North Queensland rodents. Aust. vet. $J ., 1966,42: 5-8$.

5. JONES (L. M.). Report of the International Committee on Nomenclature of Bacteria by the Subcommittee on Taxonomy of Brucella. Minutes of meeting, July 1966. Int. J. System. Bacteriol., 1967, $17: 371-375$.

6. LENNETTE (E. H.), SPAULDING (E. H.), TRUANT (J. P.). Manual of clinical microbılogy. Washington, American Society for Microbiology, 1974.

7. LUCHSINGER (D. W.), ANGUS (R. D.), GUE (C. S.), ANDERSON (R. K.). The utilization of Brucella abortus culturing and biotyping results in the epizootiologic investigation of bovine brucellosis. Proc. U.S. animal Hith Ass., 1973 : 85-99.

8. MEYER (M. E.), CAMERON (H. S.). Metabolic characterization of the genus Brucella. I. Statistical evaluation of the oxidative rates by which type 1 of each species can be identified. $J$. Bacteriol., 1961, 82 : 387-395.

9. MEYER (M. E.), CAMERON (H. S.). Metabolic characterization of the genus Brucella. II. Oxidative patterns of the described biotypes. J. Bacteriol., 1961, $82: 396-400$.

10. MORGAN (W. J. B.), CORBEL (M. J.). Recommendations for descnption of species and biotypes of the genus Brucella. Develop. biol. Siandard, 31 : $27-$ 37. Basel, S. Karger, 1976.

11. PHILIPPON (A.). Métabolisme oxydatif et lysotypie des Brucella. Symp. Ser. Immunobiol. Stand, 1970, $12: 181-190$.

12. REST (R. F.), ROBERTSON (D. C.), Characterization of the electron transport system in Brucella abortus. J. Bacteriol., 1975, 122 : 139-144.

13. RICHARDSON (M.). Cytochrome oxidase in cells of the genus Brucella. J. Bacteriol., 1975, 74 : 699-706. 
14. SMITH (L.). Cytochrome systems in aerobic electron transport. In: GUNSALUS (I. C.), STANIER (R. Y.). The bacteria. Vol. II : 365-396. New York and London, Academic Press, 1961.

15. STABLEFORTH (A. W.), JONES (L. M.). Report of the Subcommittee on Taxonomy of the genus Brucella. Int. Bull. Bacteriol. Nomen. Taxon., 1963, 13 : $145-158$.

16. STANIER (R. Y.), PALLERONI (N. J.), DOUDOROFF (M.). The aerobic Pseudomonads : a taxonomic study. J. gen. Microbiol., 1966, 43 : 159-271.

17. STEEL (K. J.). The oxidase reaction as a taxonomic stool. J. gen. Microbtol., 1961, 25 : 297-306.

18. THIMM (B.), WUNDT (W.). The epidemiological situation of brucellosis in Africa. Develop. biol. Standard., 31 : 201-217. Basel, S. Karger, 1976.

19. UMBREIT (W. W.), BURRIS (R, H.), STAUFFER (J. F.). Manometric techniques. A manual describing methods applicable to the study of tissue metabolism. Minneapolıs, Burges Publ. Co., 1964.
20. VERGER (J. M.). Un marqueur épidémiologique : la perte de l'activité oxydative d'une souche de $B$. abortus biotype $\mathrm{I}$ sur la L-asparagine par conservation à $T 4^{\circ} \mathrm{C}$ des suspensions cellulaires. Annls Rech. vét., 1973, $4: 241-252$.

21. VERGER (J. M.), GATE (M.), PIECHAUD (M.), CHATELAIN (R.), RAMISSE (J.), BLANCOU (J.). Isolement de Brucella suis biotype 5 à Madagascar, chez une chienne. Validité du nom d'espèce Brucella canis. Annls Microbiol., Inst. Pasteur, 1975, 126A: 57-74.

22. VERGER (J. M.), GRAYON (M.). Oxidative profiles of Brucella species. Ann. Sclavo., 1977, 19: $45-60$.

23. VERSHILOVA (P. A.), DRANOVSKAYA (E. A.), KUSHNAREV (V. M.). Dopolnityel'nyy sposob opryedyclyeniya prınadlyeznosti backteriy $\mathrm{k}$ rodu Brucella. (An additional method of determining the relationship of bacteria to the genus Brucella.) $Z h$. Microbiol., Mosk., 1972, 49 : 98-101. 